\title{
Validation of Recursive Partitioning Analysis and Diagnosis-Specific Graded Prognostic Assessment in patients treated initially with radiosurgery alone
}

\author{
Clinical article
}

\author{
Anna Likhacheva, M.D., ${ }^{1}$ Chelsea C. Pinnix, M.D., Ph.D., ${ }^{1}$ Neil Parikh, B.A., ${ }^{2}$ \\ Pamela K. Allen, Ph.D., ${ }^{1}$ Nandita Guha-Thakurta, M.D., ${ }^{3}$ Mary McAleer, M.D., Ph.D., ${ }^{1}$ \\ Erik P. Sulman, M.D., Ph.D., ${ }^{1}$ Anita Mahajan, M.D., ${ }^{1}$ Almon Shiu, Ph.D., ${ }^{4}$ \\ Dershan Luo, Ph.D. ${ }^{4}$ Max Chiu, B.S., ${ }^{1}$ Paul D. Brown, M.D., ${ }^{1}$ \\ Sujit S. Prabhu, M.D., F.R.C.S., ${ }^{5}$ and Eric L. Chang, M.D. ${ }^{1}$
}

Departments of ${ }^{1}$ Radiation Oncology, ${ }^{3}$ Radiology, ${ }^{4}$ Radiation Physics, and ${ }^{5}$ Neurosurgery, University of Texas MD Anderson Cancer Center; and ${ }^{2}$ Baylor College of Medicine, Houston, Texas; and ${ }^{6}$ Department of Radiation Oncology, University of Southern California, Los Angeles, California

\begin{abstract}
Object. Brain metastases present a therapeutic challenge because patients with metastatic cancers live longer now than in the recent past due to systemic therapies that, while effective, may not penetrate the blood-brain barrier. In the present study the authors sought to validate the Diagnosis-Specific Graded Prognostic Assessment (DS-GPA), a new prognostic index that takes into account the histological characteristics of the primary tumor, and the Radiation Therapy Ontology Group Recursive Partitioning Analysis (RPA) system by using a single-institution database of patients who were treated initially with stereotactic radiosurgery (SRS) alone for brain metastases.

Methods. Investigators retrospectively identified adult patients who had undergone SRS at a single institution, MD Anderson Cancer Center, for initial treatment of brain metastases between 2003 and 2010 but excluded those who had undergone craniotomy and/or whole-brain radiation therapy at an earlier time; the final number was 251 . The Leksell Gamma Knife was used to treat 223 patients, and a linear accelerator was used to treat 28 patients. The patient population was grouped according to DS-GPA scores as follows: 0-0.5 (7 patients), 1 (33 patients), 1.5 (25 patients), 2 (63 patients), 2.5 (14 patients), 3 (68 patients), and 3.5-4 (41 patients). The same patients were also grouped according to RPA classes: 1 (24 patients), 2 (216 patients), and 3 (11 patients). The most common histological diagnoses were non-small cell lung cancer (34\%), melanoma (29\%), and breast carcinoma (16\%). The median number of lesions was 2 (range 1-9) and the median total tumor volume was $0.9 \mathrm{~cm}^{3}$ (range $\left.0.3-22.9 \mathrm{~cm}^{3}\right)$. The median radiation dose was 20 Gy (range 14-24 Gy). Stereotactic radiosurgery was performed as the sole treatment (62\% of patients) or combined with a salvage treatment consisting of SRS $(22 \%)$, whole-brain radiation therapy (12\%), or resection (4\%). The median duration of follow-up was 9.4 months.

Results. In this patient group the median overall survival was 11.1 months. The DS-GPA prognostic index divided patients into prognostically significant groups. Median survival times were 2.8 months for DS-GPA Scores 0-0.5, 3.9 months for Score 1, 6.6 months for Score 1.5, 12.9 months for Score 2, 11.9 months for Score 2.5, 12.2 months for Score 3, and 31.4 months for Scores 3.5-4 ( $<<0.0001)$. In the RPA groups, the median overall survival times were 38.8 months for Class 1, 9.4 months for Class 2 , and 2.8 months for Class 3 ( $p<0.0001$ ). Neither the RPA class nor the DS-GPA score was prognostic for local tumor control or new lesion-free survival. A multivariate analysis revealed that patient age $>60$ years, Karnofsky Performance Scale score $\leq 80 \%$, and total lesion volume $>2$ $\mathrm{cm}^{3}$ were significant adverse prognostic factors for overall survival.

Conclusions. Application of the DS-GPA to a database of patients with brain metastases who were treated with SRS appears to be valid and offers additional prognostic refinement over that provided by the RPA. The DS-GPA may also allow for improved selection of patients to undergo initial SRS alone and should be studied further.
\end{abstract}

(http://thejns.org/doi/abs/10.3171/2012.3.GKS1289)

\section{KeY Words • stereotactic radiosurgery - Gamma Knife surgery • brain metastasis - prognostic factor • Diagnosis-Specific Graded Prognostic Assessment Recursive Partitioning Analysis}

Abbreviations used in this paper: DS-GPA = Diagnosis-Specific Graded Prognostic Assessment; KPS = Karnofsky Performance Scale; RPA = Recursive Partitioning Analysis; RTOG = Radiation Therapy Oncology Group; SRS = stereotactic radiosurgery; WBRT $=$ whole-brain radiation therapy.
$\mathrm{B}$ RAIN metastases arise in $10 \%-40 \%$ of patients with cancer and are 4- to 5-fold more common than primary intracranial neoplasms. ${ }^{9,12}$ The rising incidence of these lesions can be attributed to the aging population, improved neuroimaging techniques, and systemic therapies that, while effective, may not penetrate 


\section{RPA and DS-GPA in patients treated initially with SRS alone}

the blood-brain barrier. ${ }^{11}$ Aside from the mortality associated with brain metastases, the neurocognitive sequelae of these lesions contribute considerably to morbidity.

Metastatic brain disease is a heterogeneous group in both its natural history and propensity for recurrence. $\mathrm{Be}$ havior of metastatic brain cancer depends on many factors, including status of the patient's extracranial disease, number of metastatic lesions, tumor size, histological type of tumor, and many other factors. ${ }^{8,11}$ Although median survival for all patients is less than 1 year, the range can be broad, from 1 month to 2 years. ${ }^{27}$ Therefore, it is critical to select the appropriate therapy for individual patients carefully to avoid unnecessary risk and cost to those unlikely to benefit from aggressive therapy.

Management of brain metastases requires a multidisciplinary approach, with contributions from neurosurgery, radiation oncology, medical oncology, and palliative care specialties. Management options for brain metastases vary and include open brain surgery, SRS, WBRT, and supportive care. Whole-brain radiation therapy remains the mainstay of treatment. ${ }^{17}$ Combined with SRS, WBRT has been shown to improve survival in patients with a single metastasis. ${ }^{2}$ Omission of WBRT results in inferior control of distant lesions, but not necessarily worse survival. ${ }^{3,13}$ Disadvantages of WBRT include a treatment time of 1-3 weeks, preclusion of concurrent chemotherapy, and potential neurocognitive decline in long-term survivors. ${ }^{5}$

Stereotactic radiosurgery is being explored as an alternative method for initial disease control. Although management of newly diagnosed brain metastases with SRS alone does not represent a standard of care, its increasing use has been driven by both patients and physicians who fear the potential neurocognitive decline associated with WBRT and are averse to its long duration of treatment. Stereotactic radiosurgery allows for delivery of a precisely localized single dose of radiation while sparing surrounding normal brain tissue.$^{14}$ It offers local disease control comparable to that of surgery with the benefit of a more favorable side-effect profile. , $^{1,4,17,18,31}$ The rapidity with which SRS can be performed often affords patients the ability to proceed with systemic treatments in a timelier manner.

In an attempt to offer guidance for patients in whom initial treatment with SRS arises as an option, we assessed the robustness of two commonly used prognostic indices. Recursive Partitioning Analysis is one of the first and simplest tools available for estimating survival in patients with newly diagnosed brain metastases. It incorporates patient age, KPS score, status of the primary tumor, and extent of extracranial disease into a model for predicting prognosis in these patients. ${ }^{7}$ It was developed through an analysis of 1200 patients treated in 3 RTOG WBRT studies. In the RPA prognostic index, the histological type of tumor is not a statistically significant predictor of outcome.

Another widely used index is the GPA, which is based on 4 criteria: patient age, KPS score, number of brain metastases, and status of extracranial disease. ${ }^{26}$ Each of the 4 criteria is given a score of $0,0.5$, or 1.0 , which are then summed to determine the total GPA score. A GPA score of 4.0 corresponds to the best prognosis.
The authors established this index based on findings in 1960 patients, who constituted $56 \%$ of patients treated in 5 RTOG WBRT studies. Only 1 of the 5 studies included an arm in which patients underwent SRS in addition to WBRT.

Recently, a multiinstitutional analysis was performed to refine the GPA, making it specific to the histological characteristics of particular brain metastases. ${ }^{27}$ The analysis included 4259 patients with brain metastases treated with surgery, WBRT, SRS, or various treatment combinations. ${ }^{27}$ The new DS-GPA takes into account the same criteria as the GPA but defines itself according to statistically significant prognostic factors for each individual diagnosis. ${ }^{26,27}$ The DS-GPA has been touted as more quantitative and less subjective than the RPA system. ${ }^{25}$ The 6 distinct diagnoses in the DS-GPA (non-small cell and small cell lung cancer, melanoma, renal cell cancer, breast cancer, and gastrointestinal cancer) comprise a tool for future clinical trials in which patients of similar prognosis can be stratified together and treatment effects can be more easily discerned. ${ }^{25}$

In the present study we explored the prognostic value of the RPA and the DS-GPA in a cohort of patients initially treated at MD Anderson Cancer Center with SRS. We chose to focus on the DS-GPA rather than the GPA because emerging evidence has demonstrated that the former method is more accurate for determining prognosis. ${ }^{28}$ Moreover, the latest American Society for Radiation Oncology evidence-based guidelines affirm a preference for histology-specific prognostic indices. ${ }^{29}$ Our goal was to assess the value of these indices in a selection of patients with a relatively good prognosis for whom SRS as a single modality may be beneficial as opposed to those whose short expected survival would call for more traditional therapies such as WBRT.

\section{Methods}

After approval had been obtained from the institutional review board at MD Anderson Cancer Center, we retrospectively identified all patients at that institution who had undergone Gamma Knife surgery as the initial treatment or following linear accelerator-based SRS for brain metastases between June 2009 and March 2010. Patients were included if they received SRS as the initial therapy for newly diagnosed brain metastases. All histological tumor types and performance scores were allowed. No limit was set for the number of metastases treated by SRS. Patients were excluded if they had undergone previous craniotomy and/or WBRT.

\section{Radiosurgical Technique}

Patients who underwent SRS after June 24, 2009 were treated with the Leksell Gamma Knife Perfexion. The radiosurgery technique has been previously described. ${ }^{21}$ Candidates for radiosurgery were discussed in a weekly multidisciplinary conference in which members of neurosurgery, radiation oncology, and neuroradiology teams were present. Most eligible patients had 3 or fewer brain metastases at the time of the radiation oncology consultation and had an Eastern Cooperative Oncology Group 
performance score ${ }^{16}$ of $0-1$. On the day of treatment, patients underwent thin-slice (1-mm) gadolinium-enhanced MR imaging; the results were interpreted by a neuroradiologist and used for planning purposes. If a significant number of new lesions was discovered, the planned SRS was aborted after a discussion about prognosis with the patient and family. No exact cutoff was used, but a common upper limit for the number of lesions to be treated was 20 . For the majority of patients, the volume of the lesion determined the prescription dose. ${ }^{23}$ In most cases, the prescription isodose contour was the $50 \%$ isodose line. All patients were discharged home the same day.

Twenty-eight patients who fit the inclusion criteria had previously been treated at our department between February 2003 and June 2009 by linear acceleratorbased SRS, which was our department's primary delivery method for SRS before June 24, 2009. This has also been previously described. ${ }^{5}$ In these cases, a planning CT study was obtained, and at most 3 lesions were targeted at once.

\section{Follow-Up and Treatment Response Assessment}

The standard follow-up protocol required reexamination every 3 months, with contrast-enhanced neuroimaging performed during every visit. If a local or distant appearance of new lesions was diagnosed on imaging studies, the most appropriate salvage modality-resection, repeat SRS, or WBRT - was recommended. In general, resection was recommended for recurrences that were symptomatic. In our radiation department, repeat SRS was usually used in patients having a favorable prognosis, whereas WBRT was reserved for patients with multiple recurrences or a poor prognosis given the status of their extracranial disease. A small proportion of patients were lost to follow-up. In that case, the last day of face-to-face or telephone interaction was considered the last follow-up visit.

\section{Validation of Prognostic Indices}

Recursive Partitioning Analysis has been previously described. ${ }^{7}$ Briefly, 4 clinical parameters (patient age, KPS score, control of primary disease, and status of extracranial disease) were used to place patients in one of the three RPA groups, in which RPA Class 1 portends the best survival and RPA Class 3 portends the worst.

The DS-GPA was recently developed to predict survival based on the histological diagnosis of the primary cancer. ${ }^{27}$ As opposed to RPA, a slightly different set of clinical parameters (patient age, KPS score, status of extracranial disease, and number of brain metastases) is used to calculate a prognostic score. The histological type of primary tumor determines which of these parameters are used to influence the total score, which ranges from 0 to 4 , in which 0 portends the worst and 4 the best prognosis.

\section{End Points and Statistical Analyses}

Statistical comparisons were made using the Student t-test when data were normally distributed. When both dependent and independent variables were categorical, we used the Pearson chi-square test. Differences in survival within the DS-GPA and RPA prognostic groupings were analyzed with the aid of the log-rank test, and survival is presented in this paper as Kaplan-Meier plots. In addition, univariate and multivariate analyses were performed using Cox regression techniques to examine potential interactions among the entered covariates. The Wald test was used to assess the role of covariates in the model. Variables included in the models were patient age, KPS score, number of brain metastases treated, patient sex, and status of the extracranial disease. Statistical significance was set at $\mathrm{p} \leq 0.05$, and all tests were 2 -sided. All analyses were performed using Stata/MP, version 12.0 (Stata 10.1 for Windows; StataCorp).

\section{Results}

\section{Patient Characteristics}

Two hundred fifty-one patients underwent some form of SRS as initial treatment for newly diagnosed brain metastases at MD Anderson Cancer Center between February 2003 and March 2010 (Table 1). Patient characteristics showed an approximately even split between sexes. The most common tumor histological types were nonsmall cell lung cancer (34\%), melanoma (29\%), and breast carcinoma (16\%). The median number of brain metastases at diagnosis was 2 (range 1-9). The number of brain metastatic lesions was 3 or fewer in 218 patients and 4 or more in 33 patients. The median total tumor volume was $0.9 \mathrm{~cm}^{3}$ (range $0.3-22.9 \mathrm{~cm}^{3}$ ), and the median peripheral dose used in treatment was 20 Gy (range 14-24 Gy). The median follow-up duration was 9.4 months. Stereotactic radiosurgery was used as the sole treatment $(62 \%$ of patients) or in combination with salvage treatment consisting of SRS (22\%), WBRT (12\%), or resection (4\%).

\section{Patient Outcomes}

In this group of 251 patients, the median overall survival was 11.1 months ( $47 \%$ at 12 months). The median new lesion-free survival was 10 months $(45 \%$ at 12 months). Local tumor control was $95 \%$ at 12 months.

\section{Prognostic Factors}

When applied to our patients, the RPA and DS-GPA indices split the data set into prognostically different groups with respect to overall survival but not new brain lesion-free survival or local control of disease. In addition to these prognostic indices, total tumor volume ( $p$ $<0.001), \geq 3$ brain metastases $(p=0.003)$, patient age $\geq$ 60 years $(p=0.004)$, baseline KPS score $(p<0.001)$, and presence of extracranial disease $(p<0.001)$ were statistically significant predictors of overall survival in the univariate analysis. The multivariate analysis revealed that patient age $\geq 60$ years $(\mathrm{p}=0.002)$, a KPS score $\leq 80 \%$ (p $=0.005)$, and total tumor volume $>2 \mathrm{~cm}^{3}(\mathrm{p}=0.002)$ were significant adverse prognostic factors for overall survival.

\section{$R P A$}

In the RPA groups, the median overall survival was 38.8 months for Class 1, 9.4 months for Class 2, and 2.8 
TABLE 1: Characteristics of 251 patients treated with upfront SRS*

\begin{tabular}{|c|c|}
\hline Variable & No. of Patients (\%) \\
\hline male sex & $121(48)$ \\
\hline median age in yrs (range) & $59.6(15.7-93)$ \\
\hline \multicolumn{2}{|l|}{ primary tumor } \\
\hline non-small cell lung & $85(34)$ \\
\hline melanoma & $74(29)$ \\
\hline breast carcinoma & $40(16)$ \\
\hline renal cell carcinoma & $19(8)$ \\
\hline other & $33(13)$ \\
\hline \multicolumn{2}{|l|}{ baseline RPA class } \\
\hline 1 & $24(10)$ \\
\hline 2 & $216(86)$ \\
\hline 3 & $11(4)$ \\
\hline \multicolumn{2}{|l|}{ baseline DS-GPA score } \\
\hline $0-0.5$ & $7(3)$ \\
\hline 1 & $33(13)$ \\
\hline 1.5 & $25(10)$ \\
\hline 2 & $63(25)$ \\
\hline 2.5 & $14(6)$ \\
\hline 3 & $68(27)$ \\
\hline $3.5-4$ & $41(16)$ \\
\hline $\begin{array}{l}\text { median total brain metastasis vol in } \mathrm{cm}^{3} \\
\text { (range) }\end{array}$ & $0.9(0.3-22.9)$ \\
\hline median no. of brain metastases (range) & $2(1-9)$ \\
\hline 1 & $104(41)$ \\
\hline $2-3$ & $112(45)$ \\
\hline$>3$ & $35(14)$ \\
\hline \multicolumn{2}{|l|}{ SRS treatment } \\
\hline Gamma Knife surgery & $223(89)$ \\
\hline LINAC-based radiosurgery & $28(11)$ \\
\hline \multicolumn{2}{|l|}{ salvage treatment } \\
\hline none & $155(62)$ \\
\hline SRS & $56(22)$ \\
\hline WBRT & $31(12)$ \\
\hline neurosurgery alone & $4(2)$ \\
\hline neurosurgery \& SRS & $3(1)$ \\
\hline neurosurgery \& WBRT & $1(<1)$ \\
\hline
\end{tabular}

* Values represent number of patients (\%) unless otherwise indicated.

months for Class 3. A Kaplan-Meier plot representing this is shown in Fig. 1. An overall significant difference between groups $(p<0.0001)$ is evident. The median new lesion-free survival was 10.8 months for Class 1,10 months for Class 2, and 3.3 months for Class $3(\mathrm{p}=0.12)$. The median local disease control was not reached by all three classes.

\section{$D S-G P A$}

In the DS-GPA groups, the median overall survival was 2.8 months for $\leq 0.05$ points, 3.9 months for 1 point,

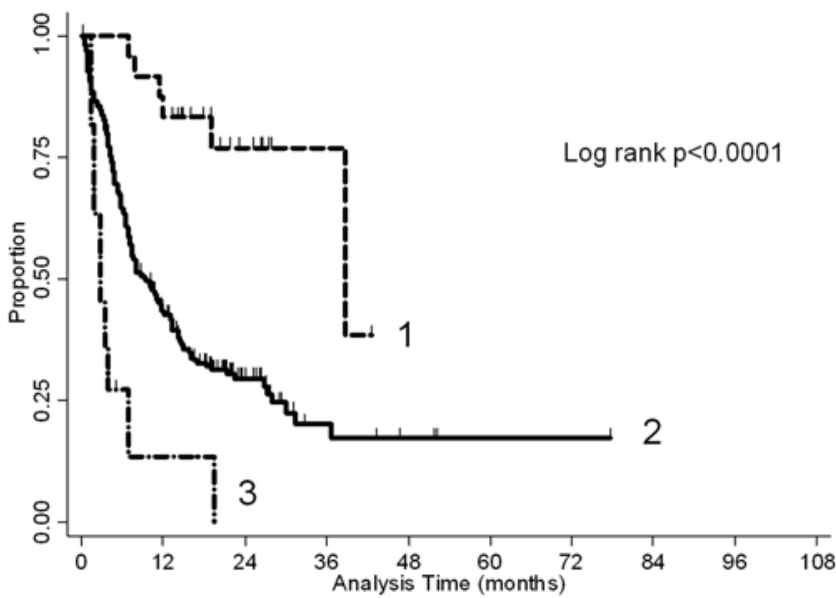

FIG. 1. Kaplan-Meier plot of overall survival stratified by RPA class.

6.6 months for 1.5 points, 12.9 months for 2 points, 11.9 months for 2.5 points, 12.2 months for 3 points, and 31.4 months for $3.5-4$ points $(\mathrm{p} \leq 0.0001)$. A Kaplan-Meier plot summarizing this is given in Fig. 2. The DS-GPA prognostic score failed to predict new brain metastasisfree survival $(\mathrm{p}=0.72)$. As with the RPA, the median local disease control was not reached by any prognostic group.

\section{Discussion}

The heterogeneity of patients with brain metastases makes prognostication challenging. Moreover, the availability of a wide variety of treatment options and combinations makes it difficult for the clinician to strike a balance between undertreatment and overtreatment in patients for whom quality and quantity of life become increasingly vital. In the present study we tested the validity of two existing prognostic indices, RPA and DS-GPA, when applied to a series of patients treated initially with SRS alone for newly diagnosed brain metastases.

A number of prognostic systems for patients with brain metastases have been developed.,10,15,26,27 The RTOG RPA and the recently developed DS-GPA are representative prognostic systems, with the former being one of the oldest systems and the latter being the most recent and refined. ${ }^{7,27}$ Both of these indices were developed using data from patients treated with WBRT. As far as we know, this is the first study to specifically validate these prognostic tools for patients in whom SRS alone was used to treat newly diagnosed brain metastases. ${ }^{6}$

The RTOG RPA index is a relatively simple system to use and was among the first indices to stratify patients treated for brain metastases into distinct prognostic groups. In the present report we demonstrate that the RTOG RPA index is valid for patients with newly diagnosed brain metastases who are treated with SRS alone. In our study, the median survival of patients was 38.8 months for RPA Class 1,9.4 months for RPA Class 2, and 2.8 months for Class 3, which represents a statistically significant difference $(\mathrm{p}<0.0001)$. Survival in each prognostic group is similar to what has been recently reported 


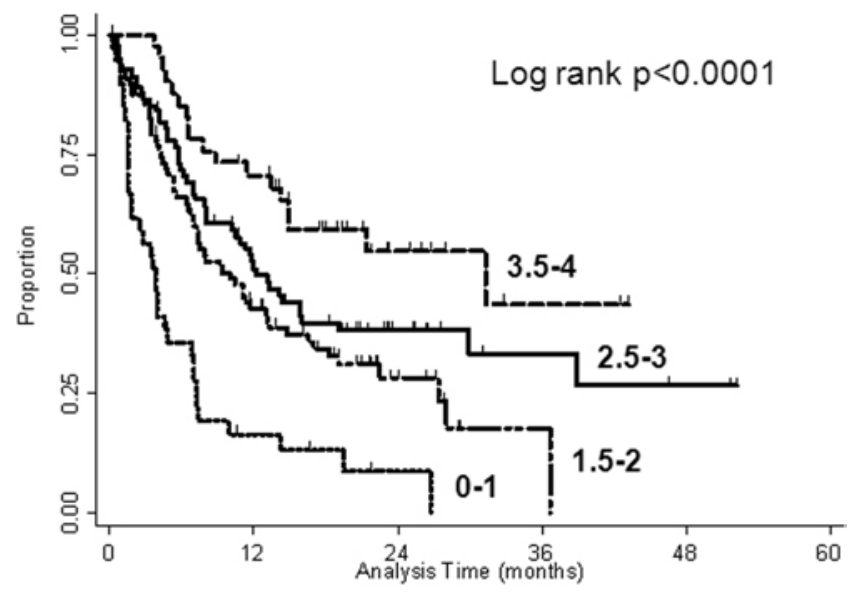

FIG. 2. Kaplan-Meier plot of overall survival stratified by DS-GPA score.

in the literature. ${ }^{15,22}$ The longer median survival by RPA groups in our population compared with the original publication ${ }^{7}$ does not imply superiority of treatment with initial SRS alone. Rather, the longer survival is most likely caused by a selection bias and possibly in part due to a more aggressive approach with improved supportive care.

The classic pattern of RPA risk stratification into highly favorable (Class 1), largely heterogeneous (Class 2 ), and highly unfavorable (Class 3 ) groups is seen in our population of patients. Although the RPA system has been criticized for its crude stratification and the heterogeneity of Class 2 , we propose that this can actually be advantageous when considering treatment with initial SRS alone. In our study, the median survival for patients in RPA Class 1 was almost 40 months, leading to a conclusion that patients who fit this category may benefit from SRS alone and thus can avoid the neurocognitive toll of WBRT. On the opposite end of the spectrum were RPA Class 3 patients with a median survival of less than 3 months. In our opinion, use of SRS should be eschewed for patients facing such a short expected survival $(<3$ months).

The fact that RPA was not effective at predicting local control and new metastasis-free survival should not be a surprise. This finding is consistent with earlier reports that tumor volume is the main predictor for local treatment failure after SRS, whereas the number of brain metastases and the histological characteristics of the tumor are predictive of no new lesions on subsequent scans. ${ }^{24,30}$

Being one of the newest prognostic indices, DS-GPA has been used and validated to a much lesser extent than the RPA. Its specificity to diagnosis ought to give this index a predictive power lost in the heterogeneity of RPA Class 2. We did find that it was valid and divided our patient population into prognostically meaningful groups. Median survival was 31.4 months for DS-GPA Scores 3.5-4, 12.2 months for Score 3, 11.9 months for Score 2.5, 12.9 months for Score 2, 6.6 months for Score 1.5, 3.9 months for Score 1, and 2.8 months for Scores $0-0.5$ (p $<0.0001$ ). The median survival for patients in each category is slightly higher than that reported in the original publication, ${ }^{27}$ where DS-GPA Scores 3.5-4 corresponded to 14.8 months, Score 3.0 corresponded to 11.6 months, Scores 1.5-2.5 corresponded to 6.4 months, and Scores 0-1.0 corresponded with 3.43 months. As mentioned earlier, this finding likely reflects a selection bias and the aggressive management of brain metastases patients at our institution. Regarding the appearance of new lesions on later imaging, this index was not effective at prognostication despite the results of previous studies that showed that the number of brain metastases and the histological diagnosis are important factors. ${ }^{24}$

In the univariate analysis, several factors-older patient age, lower KPS score, greater number of brain lesions, greater volume of brain lesions, and presence of extracranial disease-were associated with poorer survival. In the multivariate analysis, only patient age and total treatment volume were significantly associated with survival. These risk factors have been confirmed to be important predictors in numerous prospective and retrospective studies. ${ }^{15,19,20,22}$ Volume of the largest lesion is a prognostic factor in the Score Index for Radiosurgery in patients treated as part of the overall management of brain metastases. ${ }^{32}$ The reason for the importance of this patient characteristic is, in itself, an interesting question to ponder and warrants further investigation.

It appears that regardless of treatment modality-initial WBRT or initial SRS-RPA and DS-GPA indices can be used to adequately predict survival in patients with newly diagnosed brain metastases. This finding is consistent with the results of several randomized trials, in which addition of WBRT to SRS improved future control of brain disease but not survival. ${ }^{3,13}$ One retrospective series found that the particular radiation regimen (SRS alone or WBRT alone) was not predictive of survival in patients in RPA Classes 1 and 2 with 1-3 brain metastases. ${ }^{20}$ This can be explained in part by the success of salvage treatment. For example, $38 \%$ of patients in our study received additional treatment after SRS (12\% underwent WBRT). This observation may also suggest that patient survival is heavily determined by underlying biological factors rather than by choice of therapy.

There are several limitations to this study. Its retrospective nature implies shortcomings inherent in every study of this type. Of these, the most obvious weakness is the selection bias for initial SRS, which can certainly account for the longer overall survival in our patients compared with that in historical controls. Survival may also be inflated by the use of salvage therapy. Its use dilutes the significance of these two indices when applied to patients who receive initial SRS as the primary therapy. In addition, the external validity of our results will depend on patient populations elsewhere, since referral practices and treatment standards for patients with brain metastases may vary by institution.

\section{Conclusions}

Both RPA classes and DS-GPA scores have prognostic value in a population of patients treated initially with SRS alone. Nevertheless, these indices did not predict local control of the disease or future emergence of lesions 
on imaging. For this patient population, patient age and lesion volume predicted mortality in the multivariate analysis. Prognostic indices appear to be valid in predicting survival in patients with brain metastases regardless of the treatment modality used. They can serve as a useful tool for clinicians who need to balance the risks of over- and undertreatment. Although these indices provide guidance, decision making must be done on an individual basis.

\section{Disclosure}

This research was supported in part by the National Institutes of Health through MD Anderson's Cancer Center Support Grant CA016672. Dr. Likhacheva received a travel grant from Elekta to travel to the 16th Annual Leksell Gamma Knife Society Meeting. Otherwise, the authors report no conflict of interest concerning the materials or methods used in this study or the findings specified in this paper.

Author contributions to the study and manuscript preparation include the following. Conception and design: Chang, Likhacheva, Pinnix. Acquisition of data: Likhacheva, Pinnix, Parikh, McAleer, Sulman, Mahajan, Chiu, Prabhu. Analysis and interpretation of data: Chang, Likhacheva. Drafting the article: Likhacheva. Critically revising the article: all authors. Reviewed submitted version of manuscript: all authors. Approved the final version of the manuscript on behalf of all authors: Chang. Statistical analysis: Allen. Administrative/technical/material support: Guha-Thakurta, McAleer, Sulman, Mahajan, Shiu, Luo, Brown. Study supervision: Chang, Brown.

\section{References}

1. Adler JR, Cox RS, Kaplan I, Martin DP: Stereotactic radiosurgical treatment of brain metastases. J Neurosurg 76:444449, 1992

2. Andrews DW, Scott CB, Sperduto PW, Flanders AE, Gaspar LE, Schell MC, et al: Whole brain radiation therapy with or without stereotactic radiosurgery boost for patients with one to three brain metastases: phase III results of the RTOG 9508 randomised trial. Lancet 363:1665-1672, 2004

3. Aoyama H, Shirato H, Tago M, Nakagawa K, Toyoda T, Hatano K, et al: Stereotactic radiosurgery plus whole-brain radiation therapy vs stereotactic radiosurgery alone for treatment of brain metastases: a randomized controlled trial. JAMA 295:2483-2491, 2006

4. Auchter RM, Lamond JP, Alexander E, Buatti JM, Chappell R, Friedman WA, et al: A multiinstitutional outcome and prognostic factor analysis of radiosurgery for resectable single brain metastasis. Int J Radiat Oncol Biol Phys 35:27-35, 1996

5. Chang EL, Wefel JS, Hess KR, Allen PK, Lang FF, Kornguth DG, et al: Neurocognition in patients with brain metastases treated with radiosurgery or radiosurgery plus whole-brain irradiation: a randomised controlled trial. Lancet Oncol 10: 1037-1044, 2009

6. Chidel MA, Suh JH, Reddy CA, Chao ST, Lundbeck MF, Barnett GH: Application of recursive partitioning analysis and evaluation of the use of whole brain radiation among patients treated with stereotactic radiosurgery for newly diagnosed brain metastases. Int J Radiat Oncol Biol Phys 47:993-999, 2000

7. Gaspar L, Scott C, Rotman M, Asbell S, Phillips T, Wasserman T, et al: Recursive partitioning analysis (RPA) of prognostic factors in three Radiation Therapy Oncology Group (RTOG) brain metastases trials. Int J Radiat Oncol Biol Phys 37:745-751, 1997

8. Gaspar LE, Mehta MP, Patchell RA, Burri SH, Robinson PD,
Morris RE, et al: The role of whole brain radiation therapy in the management of newly diagnosed brain metastases: a systematic review and evidence-based clinical practice guideline. J Neurooncol 96:17-32, 2010

9. Gavrilovic IT, Posner JB: Brain metastases: epidemiology and pathophysiology. J Neurooncol 75:5-14, 2005

10. Golden DW, Lamborn KR, McDermott MW, Kunwar S, Wara WM, Nakamura JL, et al: Prognostic factors and grading systems for overall survival in patients treated with radiosurgery for brain metastases: variation by primary site. J Neurosurg 109 Suppl:77-86, 2008

11. Jenkinson MD, Haylock B, Shenoy A, Husband D, Javadpour M: Management of cerebral metastasis: evidence-based approach for surgery, stereotactic radiosurgery and radiotherapy. Eur J Cancer 47:649-655, 2011

12. Kalkanis SN, Linskey ME: Evidence-based clinical practice parameter guidelines for the treatment of patients with metastatic brain tumors: introduction. J Neurooncol 96:7-10, 2010

13. Kocher M, Soffietti R, Abacioglu U, Villà S, Fauchon F, Baumert BG, et al: Adjuvant whole-brain radiotherapy versus observation after radiosurgery or surgical resection of one to three cerebral metastases: results of the EORTC 22952-26001 study. J Clin Oncol 29:134-141, 2011

14. Loeffler JS, Shrieve DC, Wen PY, Fine HA, Kooy HM, Addesa AE, et al: Radiosurgery for intracranial malignancies. Semin Radiat Oncol 5:225-234, 1995

15. Lorenzoni J, Devriendt D, Massager N, David P, Ruíz S, Vanderlinden $\mathrm{B}$, et al: Radiosurgery for treatment of brain metastases: estimation of patient eligibility using three stratification systems. Int J Radiat Oncol Biol Phys 60:218-224, 2004

16. Oken MM, Creech RH, Tormey DC, Horton J, Davis TE, McFadden ET, et al: Toxicity and response criteria of the Eastern Cooperative Oncology Group. Am J Clin Oncol 5:649-655, 1982

17. Patchell RA, Tibbs PA, Regine WF, Dempsey RJ, Mohiuddin M, Kryscio RJ, et al: Postoperative radiotherapy in the treatment of single metastases to the brain: a randomized trial. JAMA 280:1485-1489, 1998

18. Patchell RA, Tibbs PA, Walsh JW, Dempsey RJ, Maruyama Y, Kryscio RJ, et al: A randomized trial of surgery in the treatment of single metastases to the brain. N Engl J Med 322: 494-500, 1990

19. Rades D, Bohlen G, Pluemer A, Veninga T, Hanssens P, Dunst $\mathrm{J}$, et al: Stereotactic radiosurgery alone versus resection plus whole-brain radiotherapy for 1 or 2 brain metastases in recursive partitioning analysis class 1 and 2 patients. Cancer 109: 2515-2521, 2007

20. Rades D, Pluemer A, Veninga T, Hanssens P, Dunst J, Schild SE: Whole-brain radiotherapy versus stereotactic radiosurgery for patients in recursive partitioning analysis classes 1 and 2 with 1 to 3 brain metastases. Cancer 110:2285-2292, 2007

21. Régis J, Tamura M, Guillot C, Yomo S, Muraciolle X, Nagaje M, et al: Radiosurgery with the world's first fully robotized Leksell Gamma Knife PerfeXion in clinical use: a 200-patient prospective, randomized, controlled comparison with the Gamma Knife 4C. Neurosurgery 64:346-356, 2009

22. Sanghavi SN, Miranpuri SS, Chappell R, Buatti JM, Sneed PK, Suh JH, et al: Radiosurgery for patients with brain metastases: a multi-institutional analysis, stratified by the RTOG recursive partitioning analysis method. Int J Radiat Oncol Biol Phys 51:426-434, 2001

23. Shaw E, Scott C, Souhami L, Dinapoli R, Kline R, Loeffler J, et al: Single dose radiosurgical treatment of recurrent previously irradiated primary brain tumors and brain metastases: final report of RTOG protocol 90-05. Int J Radiat Oncol Biol Phys 47:291-298, 2000

24. Sheehan JP, Yen CP, Nguyen J, Rainey JA, Dassoulas K, 
Schlesinger DJ: Timing and risk factors for new brain metastasis formation in patients initially treated only with Gamma Knife surgery. Clinical article. J Neurosurg 114:763-768, 2011

25. Sperduto PW: What is your patient's GPA and why does it matter? Managing brain metastases and the cost of hope. Int J Radiat Oncol Biol Phys 77:643-644, 2010

26. Sperduto PW, Berkey B, Gaspar LE, Mehta M, Curran W: A new prognostic index and comparison to three other indices for patients with brain metastases: an analysis of 1,960 patients in the RTOG database. Int J Radiat Oncol Biol Phys 70:510-514, 2008

27. Sperduto PW, Chao ST, Sneed PK, Luo X, Suh J, Roberge $\mathrm{D}$, et al: Diagnosis-specific prognostic factors, indexes, and treatment outcomes for patients with newly diagnosed brain metastases: a multi-institutional analysis of 4,259 patients. Int J Radiat Oncol Biol Phys 77:655-661, 2010

28. Sperduto PW, Kased N, Roberge D, Xu Z, Shanley R, Luo X, et al: Summary report on the graded prognostic assessment: an accurate and facile diagnosis-specific tool to estimate survival for patients with brain metastases. J Clin Oncol 30: 419-425, 2012

29. Tsao MN, Rades D, Wirth A, Lo SS, Danielson BL, Gaspar LE, et al: Radiotherapeutic and surgical management for newly diagnosed brain metastasis(es): an American Society for Radiation Oncology evidence-based guideline. Practical Radiation [in press], 2012
30. Varlotto JM, Flickinger JC, Niranjan A, Bhatnagar AK, Kondziolka D, Lunsford LD: Analysis of tumor control and toxicity in patients who have survived at least one year after radiosurgery for brain metastases. Int J Radiat Oncol Biol Phys 57:452-464, 2003

31. Vecht CJ, Haaxma-Reiche H, Noordijk EM, Padberg GW, Voormolen JH, Hoekstra FH, et al: Treatment of single brain metastasis: radiotherapy alone or combined with neurosurgery? Ann Neurol 33:583-590, 1993

32. Weltman E, Salvajoli JV, Brandt RA, de Morais Hanriot R, Prisco FE, Cruz JC, et al: Radiosurgery for brain metastases: a score index for predicting prognosis. Int J Radiat Oncol Biol Phys 46:1155-1161, 2000

Manuscript submitted January 10, 2012.

Accepted March 13, 2012.

This report was presented at the ASTRO annual meeting in Miami, Florida, October 2-6, 2011. An updated report was presented at the 16th International Meeting of the Leksell Gamma Knife Society in Sydney, Australia, on March 27, 2012.

Please include this information when citing this paper: DOI: 10.3171/2012.3.GKS1289.

Address correspondence to: Eric L. Chang, M.D., University of Southern California, 1441 Eastlake Avenue, NOR G-356, Los Angeles, California 90033. email: Eric.Chang@ health.usc.edu. 